\title{
Effects of dietary Lasia spinosa Thw. powder supplementation on growth performance, blood metabolites, antioxidant status, intestinal morphology, and cecal microbiome in Chinese yellow-feathered broilers
}

Hong Yongxing

Guangxi University

Zhang Lang

Guangxi University

Tian Kui

Guangxi University

Sun Haodong

Guangxi University

Liu Xingting

Guangxi University

Tang Yanfei

Guangxi Fufeng Agriculture and Animal Husbandry Co Ltd

Hassanin Abdallah A.

Zagazig University

Abdelnour Sameh A.

Zagazig University

Liao Yuying

Guangxi Veterinary Research Institute

Suthikrai Wanwipa

Chulalongkorn University

Srisakwattana Kittiya

Chulalongkorn University

Tharasanit Theerawat

Chulalongkorn University

Yangqing Lu ( $\sim$ luyangqing@126.com )

Guangxi University https://orcid.org/0000-0003-1641-6142

Research 
Keywords: Chinese Yellow-feathered broiler, Lasia spinosa Thw, Growth performance, Antioxidant status, Intestinal morphology, Cecal microbiome

Posted Date: July 19th, 2021

DOI: https://doi.org/10.21203/rs.3.rs-710808/v1

License: @ (i) This work is licensed under a Creative Commons Attribution 4.0 International License. Read Full License 


\section{Abstract}

Background: Lasia spinosa Thw. (LST) has been proven to be nutritious and have growth-promoting, antioxidant functions and so on, but its effect in chicken is still unclear. This study aimed to evaluate the effects of dietary LST powder supplementation on growth performance, blood metabolites, antioxidant status, intestinal morphology and cecal microbiome in Chinese yellow-feathered broilers.

Methods: A total of 400 one-day-old yellow-feather broilers were randomly allotted to 4 dietary treatments: LST0 group (a basal diet), LST1 group (a basal diet with 1\% LST powder), LST2 group (a basal diet with 2\% LST powder), LST4 group (a basal diet with 4\% LST powder), ten replicates for each treatment and 10 broilers in each treatment group.

Results: Results indicated that the average daily feed intake of broilers during $22-42 \mathrm{~d}$ and the average daily gain of chickens over all periods were significantly increased by dietary supplementation of LST powder compared to a control group, while the feed conversion ratio during the overall periods was markedly decreased. The levels of SOD, CAT and GSH-Px in serum, liver and breast muscle were also significantly increased in LST supplemented groups, while ROS and MDA in serum, liver and breast muscle were decreased. Furthermore, the levels of TG and LDL-C were significantly decreased by the addition of dietary LST powder, while levels of $\mathrm{HDL}-\mathrm{C}, \mathrm{Ca}, \mathrm{Fe}, \mathrm{Mg}$ and $\mathrm{P}$ were linearly increased. Regarding the gut morphometric, crypt depth was significantly decreased by LST supplementation, while villus height and the ratio of villus height to crypt depth were notably increased. Sequencing of 16S rRNA from the cecal contents of broilers revealed that the composition of the chicken gut microbiota was altered by LST supplementation. Moreover, the diversity of microbiota in broilers was increased in the LST1 groups but was decreased in the LST2 and LST4 groups compared with LST0 groups. The differential genera enriched in LST1 groups, such as Bacillus, Odoribacter, Sutterella, Anaerofilum, Peptococcus, were closely related to the increased growth performance, antioxidant status, intestinal morphology, $\mathrm{Ca}, \mathrm{Mg}$ and reduced blood lipid in the treated broilers.

Conclusions: The supplementation of LST powder to the diets of Chinese yellow-feathered broilers improved growth performance, lipid profile, antioxidant indices, intestinal morphology and gut microbiota balance, with its optimum level in yellow-feathered broilers' diet being $1 \%$.

\section{Introduction}

For several decades, the use of antibiotic growth promoters (AGP) in the livestock sector enhanced growth performance, intestinal health, feed efficiency, diseases prevention, and mitigation of the stress caused by environmental and management factors in the intensive poultry farming [1, 2]. However, with the long-term and extensive use of AGP, public concerns have augmented regarding to the resistant bacteria and antibiotic drug residues [3, 4]. For this, many countries such as the European Union [5], and recently China have completely banned the utilize of AGP as growth promoters in animal diets. Given the high demand for highquality poultry products, the development of sustainable, safe, practical and effective feed additives that can both maintain broiler health and enhance productive potential is imperative [6]. Presently, the use of herbal plants and their derivatives is gaining more attention globally for poultry production that could support in maintaining health status and growth performance [6-8]. 
Lasia spinosa Thwaites (LST) belong to the Aracea family, which cultivates in numerous areas globally including south Asia, India and China [9]. The methanolic extracts of LST have been used as inhibitor of reactive oxygen species (ROS) [10]. This may be attributed to their high contents of phenolic compounds, richness in essential microelements and pro-vitamin A carotenoids [11, 12]. Moreover, LST exhibits several biological impacts such as anti-hyperglycemic [13], anti-hyperlipidemic [12], anti-bacterial [13,14], antioxidant [15], anthelmintic [14] and anti-inflammatory activities [16], representing beneficial potency in poultry diets.

Studies have reported that LST can be used as a natural source of phytoestrogens and phytoandrogens [17]. Phenolic molecules, such as gentistic acid, apigenin, syringic acid, morin, cinnamic acid and 4-

hydroxybenzoic acid, are the main component of LST extract and have been known for their antioxidants, anti-microbial, immunomodulatory and anti-inflammatory capacity $[18,19]$. Besides, in some earlier studies, dietary inclusion of LST meal has been described to have boosted the performance production and feed efficiency in cattle, buffalo and fish [17, 20, 21]. This may attribute to an increase of the goblet cells and enhance the intestinal health in fish by LST supplementation [20]. However, to our knowledge, report on the supplementation of broiler diets with LST as an alternative to antibiotics is not seen yet. In this research, the effects of LST dietary inclusion on the growth performance, blood metabolites, antioxidant profile, intestinal morphology and cecal microbiota in broilers were evaluated, in order to increase knowledge and provide innovative information on the usage of LST in chicken feed.

\section{Materials And Methods}

The current study was conducted on the Experiment Farm of Guangxi University and Guangxi Fufeng Agriculture and Animal Husbandry Co., Ltd (Nanning, P. R. China). The bird maintenance and experimental protocols in this study were conducted following the guidelines approved by the Institutional Animal Care and Use Committee of Guangxi University (GXU2018-003).

\section{Preparation of the plant}

The rhizomes of LST were collected from Chongzuo, P. R. China. The fresh plants were washed and dried in the open air without sunlight. The dry rhizomes of LST were machined into powder and then stored at room temperature until use.

\section{Bird and experimental design}

A total of 400 one-day-old chickens (males, Chinese yellow-feathered) were individually weighed (initial body weight [IBW]: $42.52 \pm 0.06$ ) and randomly assigned into four experimental groups. The experimental groups were as the following:1) LST0 group, a basal diet; 2) LST1 group, a basal diet with 1\% LST powder; 3) LST2 group, a basal diet with 2\% LST powder; 4) LST4 group, a basal diet with 4\% LST powder. Each experimental group was replicated in 10 cages of 10 chickens with a homogeneous IBM per cage $(70 \mathrm{~cm}$-width $\times 70 \mathrm{~cm}$ length $\times 35 \mathrm{~cm}$-height). Food and water were automatically delivered ad libitum during the whole tested period. As shown in Table S1, ingredients and nutrient contents of basal diet (as fed basis) for growing broiler of 1-21d and 22-42d. Furthermore, ingredients and nutrient contents of LST were detected by Ulanqab E-more Tech Co., Ltd., and represented in Table S2. 
All chickens were maintained in an environmentally controlled chicken house. The birds were subjected to a 24-h photoperiod in the first three days, and then hours of lights were progressively reduced to 10L: 14D. The room temperature was retained at $36^{\circ} \mathrm{C}$ for the first week, and then it was decreased by $4^{\circ} \mathrm{C}$ weekly and at the 5- 6 weeks it was maintained at $22^{\circ} \mathrm{C}$. Besides, all animals were vaccinated with a Newcastle disease vaccine on the 1 th and 10th day, and a deactivated infectious bursal disease vaccine on 21 th day.

\section{Growth performance}

Chickens were individually weighed at 21 and 42 days of age to define the average daily weight gain (ADG). Feed intake ( $\mathrm{Fl}$ ) of chickens in each replicate was monitored daily and, feed conversion ratio (FCR g feed/g gain) was considered by using data of ADG and Fl. The mortality rate was recorded daily in each group. All growth parameters were considered for the periods from day 1 to day 21 , from day 22 to day 42 , and from day 1 to day 42 according to the study protocol.

\section{Sampling collection}

At the end (day 42) of the experiment, chickens were deprived of feed for $12 \mathrm{~h}$. Then 40 chickens ( 1 chicken per cage) were selected on treatment-wise average live weight to be representative, and slaughtered by cervical dislocation to collect a blood sample. The blood samples were separated for serum by centrifugation at $4^{\circ} \mathrm{C}, 3,000 \mathrm{rpm}$ for $10 \mathrm{~min}$. Serums were transferred into $1.5 \mathrm{~mL}$ microcentrifuge tubes, and finally stored at $-80^{\circ} \mathrm{C}$ for further examination. Immediately after slaughter, all segments of the small intestine, as well as cecum, were excised.

The gastrointestinal contents were collected from the cecum, hired in cryogenic vials, kept on dry ice and delivered to the laboratory and stored at $-80^{\circ} \mathrm{C}$. Cecal contents were used for the detection of microbiota composition. For the sample of histological examination, $3 \mathrm{~cm}$ of small intestinal segment was taken from the middle of the duodenum, jejunum, ileum and cecum respectively, washed in phosphate buffered saline (PBS) solution to eliminate all the content and fixed in $10 \%$ neutral buffered formalin solution (Solarbio, Beijing, P. R. China). For the assessment of the antioxidants in liver and breast muscles, tissues were collected into $1.5 \mathrm{~mL}$ Eppendorf tubes, then washed by PBS and transported to the laboratory on dry ice and frozen immediately in liquid nitrogen $\left(-80^{\circ} \mathrm{C}\right)$.

\section{Blood metabolites assessments}

Serum biochemical metabolites, including liver function indices, kidney function indices, ionic content, glucose, rheumatoid factor (RF), lipids and lipoproteins, were assessed. Serum aspartate aminotransferase (AST), alanine aminotransferase (ALT), uric acid (UA), total protein (TP), albumin (ALB), direct bilirubin (DB), total bilirubin (TB) urea, cholesterol (CHOL), rheumatoid factor (RF), triglyceride (TG), high-density lipoprotein cholesterol (HDL-C), low-density lipoprotein cholesterol (LDL-C) levels were determined spectrophotometrically using commercial kits (URIT Medical Electronic Co., Ltd., Guilin, P. R. China). All assays were conducted by a URIT-8021AVet Auto-Blood Biochemical Analyzer (URIT Medical Electronic Co., Ltd., Guilin, P. R. China) according to the manufacturer's instructions. Serum ionic levels, including Calcium (Ca), Phosphorus (P), Iron ( $\mathrm{Fe}$ ) and Magnesium (Mg), were evaluated using an atomic absorption spectrophotometer (URIT Medical Electronic Co., Ltd., Guilin, P. R. China).

\section{Antioxidant parameters analysis}


Approximately $0.1 \mathrm{~g}$ of tissue samples (liver and breast muscle) were collected and homogenized with $0.9 \mathrm{~mL}$ PBS (0.01mol/L), PH 7.4 using a TG rinder homogenizer (Tiangen Biotech CO., Beijing, P. R. China). Afterward, the supernatants were collected after centrifugation at $3000 \times \mathrm{g}$ for $20 \mathrm{~min}$ at $4^{\circ} \mathrm{C}$. The levels of malondialdehyde (MDA), reactive oxygen species (ROS), catalase (CAT), superoxide dismutase (SOD), and glutathione peroxidase (GSH-Px) in tissue (liver and muscle) samples and the serum, as well as the levels of TP in tissue samples (breast muscle and liver) were evaluated using ELISA technique (enzyme-linked immunosorbent sorbent assay) kits with a microplate reader according to manufacturer's instructions (Shanghai Enzyme linked Biotechnology Co., Ltd., Shanghai, P. R. China). All data were normalized alongside TP level in each tissue sample for inter-sample assessment.

\section{Histomorphology study}

The fixed tissues were dehydrated and fixed with paraffin in a 2T-12M tissue processor (XiaoganYaguang Medical Electronic Technology Co., Ltd., Xiaogan, P. R. China), and then embedded in paraffin blocks using an embedding system (Leica, Germany). The blocks were then sliced into $7 \mu \mathrm{m}$-thick sections using a rotary microtome (Leica, Germany) and stained with Haematoxylin \& Eosin (HE). The crypt depth (Cd, from the basis of the villus to the submucosa), villus height (Vh, from the tip of the villus to the crypt) and the villus height to crypt depth $(\mathrm{Vh} / \mathrm{Cd})$ ratio were evaluated [22]. Histomorphology analyses were performed on 6 well-oriented and intact villi and 6 crypts chosen from duodenum, jejunum, ileum and cecum. Slices were photographed

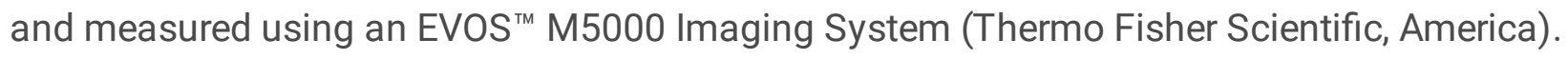

\section{Microbiome analysis}

Caecal contents (five replicates/treatment) were collected and sent to Beijing microread Technology Co., Ltd. (Beijing, P. R. China) for 16S sequencing using an Illumina HiSeq 2500 PE 250 platform (Illumina, San Diego, USA). All sequence data processing was performed using the QIIME software package (V1.9.1). Sequences were paired-end and aligned against the Green Genesdatabase (http://greengenes.lbl.gov). The Mothur software package (V1.23.1) was used to identify and remove chimeric sequences. Operational taxonomic units (OTUs) were assigned at a $97 \%$ identity using the Green Genes database. Finally, The Venn diagram with shared and unique OTUs was used to identify the similarity and differences among treatments. Alpha and beta diversity analyses were performed for differences in species composition among samples. Linear discriminant analysis (LDA) effect size (LEfSe) analysis was performed to identify differentially abundant bacterial taxa based on LDA score $>2.0$. Spearman correlation analyses were conducted to identify the relationship between physicochemical factors and intestinal flora.

\section{Statistical analysis}

Statistical analysis was performed by the IBM SPSS Statistics V23.0.0 software (SPSS Inc., Chicago, IL, USA). All data above were tested using one-way analysis of variance (ANOVA), and the effect of dietary LST powder supplementation was evaluated by means of polynomial contrasts (linear and quadratic). Significant differences among the treatments were tested using Least Significant Difference (LSD) Test. The results were expressed as the mean and standard error of the mean (SEM). $P$ values $<0.05$ were considered statistically significant. Means followed by different letters differed significantly $(P<0.05)$, followed by no or same letters indicated no significant difference. A statistical trend was considered for $0.05<P \leq 0.10$. 


\section{Results}

\section{Growth performance}

Data of the growth performance of the broiler are revealed in Table 1. Briefly, a significant increase $(P=$ 0.0002) of ADFI during 22-42d and a decrease $(P<0.0001)$ of FCR during the overall periods were observed in chickens with dietary addition of LST powder. Compared with LST0 groups, the ADG of chickens was significantly $(P<0.01)$ increased by dietary LST powder supplement during the overall periods. The highest values $(P<0.0001)$ of BW at $42 d$ were noticed in LST1, followed by both LST2 and LST4 groups. Given BW at $21 \mathrm{~d}$, no significant alterations were detected between the control and LST2 groups. The mortality rates were low in the control and tested groups (about $0.0-2.0 \%$ as a normal range) and were not influenced ( $P$ > $0.05)$ by dietary treatments

\section{Blood metabolites}

The blood metabolites and mineral profile of broiler fed the different diets for 42 days were shown in Table 2 . No significant differences were detected in all blood metabolites except for TBIL, triglyceride, HDL_C and LDL_C. The levels of TBIL were significantly decreased $(P<0.0001)$ in LST1 in compared with other treated groups. The levels of TG and LDL_C were significantly reduced $(P<0.01)$ by dietary inclusion of LST, whereas the levels of HDL_C were increased $(P<0.0001)$ in all supplemented groups. The mineral levels of $\mathrm{Ca}$, $\mathrm{Fe}$ and $P$ were significantly increased $(P<0.01)$ in groups with dietary supplementation of LST. Only birds fed with $4 \%$ exhibited the higher levels of magnesium $(P=0.0025)$ compared with the other treated and control groups.

\section{Antioxidant status}

The antioxidant profiles of serum, liver and breast muscle on broilers are presented in Table 3. The levels of GSH-Px and SOD in the liver were not statistically $(P>0.05)$ influenced by the inclusion of LST in broiler diets. Generally, compared to the control group, the levels of ROS and MDA in the serum and tissues (liver and breast muscles) of broiler, were significantly $(P<0.05)$ decreased by the diets supplement with LST. On the other hand, animals fed with dietary inclusion of LST powder had the significantly highest values of CAT, SOD, and GSH-Px in serum and tissues compared with the control.

\section{Histomorphology study}

Table 4 and Fig. 1 represent the data regarding the histological features of broiler fed with test diets for 42 days. Birds fed with different levels of LST showed greater $(P<0.05)$ villus height $(V h)(P<0.01)$ and villus height /crypt depth $(\mathrm{Vh} / \mathrm{Cd})$ of the intestinal and cecum compared with control groups. On the other hand, the crypt depth was significantly $(P<0.05)$ decreased in the intestinal and cecum of all LST treated groups as compared with the control groups.

\section{Microbiota characterization}

The Venn diagram revealed that the cecal microbiome of Chinese yellow-feathered broilers shared 1638 OTUs across the groups (Fig. 2A). The unique OTUs were 2235, 3894, 1673 and 1820 in the LST0, LST1, LST2 and LST4 groups, respectively. The results of principle component analysis (PCA) plot demonstrated that diversity 
of cecal microbiome community produced a diverse cluster (Anosim, $\mathrm{P}=0.001$ ) and separated from each group (Fig. 2B). In addition, the composition of the top 10 phyla and genera in cecal contents of Chinese yellow-feathered broilers were detected (Fig. 2C and 2D). Based on this analysis, the dominant phyla of cecal microbiota were Firmicutes, Bacteroidetes, Proteobacteria, Verrucomicrobia, Synergistetes, Deferribacteres, Cyanobacteria, Actinobacteria, Euryarchaeota. At the genus field, the dominant microbiome in cecal contents were Bacteroides, Faecalibacterium, Oscillospira, Megamonas, Helicobacter, Campylobacter, [Ruminococcus] (Fig. 2C).

PD whole tree index, Chao1 index and obeserved species index revealed that the a-diversity was significantly higher $(P<0.05)$ in LST1 groups compared with the control groups. In contrast, a-diversity in the LST2 and LST4 groups were significantly reduced $(P<0.05)$ compared with other groups. These results suggest the gut microbiota of Chinese yellow-feathered broiler was significantly altered by dietary LST supplementation.

LEfSe analysis was performed to identify specific phylotypes of the microbiome as responsive to LST supplemented into broiler diets at 42 days (Fig. 3). As shown in Fig. 3A, a total of 18 bacterial taxonomic clades showed statistical differences among all test diets. At the class level, birds fed with $1 \%$ LST had a greater $(\mathrm{P}<0.05)$ abundance of Deferribacterales and Synergistales than those fed the other test diets. At the order level, Deferribacterales, Clostridiales, Burkholderiales, Synergistales were overrepresented in the LST1 groups, while Methanomicrobiales and Pasteurellales were overrepresented in the LST2 and LST4 groups, respectively. In addition, at the family level, the relative abundances of Rikenellaceae, Odoribacteraceae, Bacillaceae, Christensenellaceae and Synergistaceae were significantly increased in the LST1 groups compared with those in other groups. The abundances of Helicobacteraceae, Methanocorpusculaceae, Peptostreptococcaceae and Pasteurellaceae were significantly increased in the LST0, LST2 and LST4 groups, respectively. LDA score computed for differential abundant taxa is presented in Fig. 3B. Totally, at the genus level, Barnesiella, Mucispirillum, Sutterella, Odoribacter, Anaerofilum, Bacillus, Alistipes and Peptococcus were enriched in the LST1 groups, while only Gallibacterium was enriched in the LST4 groups. The results further indicated that the dietary LST supplementation altered the abundance of gut microbiota compared with the control groups.

\section{Gut microbiota alteration mediate the differential regulatory effects}

Spearman correlation analysis of biological pathways was conducted to better understand the regulatory function of the gut microbiome as affected by inclusion of dietary LST. Association of differential genera with growth performance, serum parameters, antioxidant status and intestinal morphology were recognized (Table 5). The relative abundance of Alistipes was positively related to ROS of breast muscle, duodenum Cd and triglyceride, while negatively correlated with serum and hepatic CAT level and Vh/Cd of duodenum. The relative abundance of Barnesiella was positively related to serum ROS and MDA, while negatively correlated with hepatic GSH-Px. Additionally, the abundance of Mucispirillum was positively related to Ca and jejunal $\mathrm{Vh} / \mathrm{Cd}$, while negatively correlated with jejunal $\mathrm{Cd}$. The abundance of Bacillus was positively related to serum CAT and SOD, Ca, duodenal Vh and Vh/Cd, SOD and GSH-Px of breast muscle, while negatively correlated with FCR, TBIL, and LDL-C at 1-42 days. The abundance of Peptococcus was positively related to $\mathrm{Ca}$, while negatively correlated with TBIL and LDL-C. Furthermore, the relative abundance of Gallibacterium was positively related to $\mathrm{Mg}$ and LDL-C, while negatively correlated with serum ROS contents. Positive correlation 
was observed between Odoribacter and ADG and BW at1-42 days, while Sutterella was positively related to Mg level in the serum.

\section{Discussion}

In the last decades, dietary manipulation with innovative feed supplements, such as medicinal plant and its derivatives, are among the common advanced strategies [5, 24-26]. Medicinal plants are reflected a cornerstone of medicine since earliest times, and have been employed as promoters of antioxidant capacity, immunity and growth in animals [26]. Lasia spinosa Thw. (LST) has been used medicinally since ancient times for its diverse biological activities such as anti-bacterial and anti-septic properties [13-16].

In the present study, dietary LST powder supplementation significantly boosted the growth performance of the chickens involved, produced a remarkable increase in ADG and final body weight, and caused a statistical decrease in FCR when compared to the control diet, in accordance with the findings that the inclusion of LST in fish diets significantly improved the growth performance and feed utilization [20]. In addition, the current study displayed no significant difference in mortality rate was noticed in groups that received LST compared with the control, which agreed with the described by Munglue et al. [20] who perceived no significant alterations concerning survival rate in fish as a response to the dietary LST. In parallel, the supplementation of LST in buffalo had a positive impact on the growth indices [21]. However, the underlying mechanisms of the growth-promoting effect of LST are still not understood. Phesatcha et al. [2] confirmed that the inferior FCR and boosted growth may relate to enhanced appetite and digestion in beef-cattle fed diets enriched with LST $(60-90 \mathrm{~g} /$ day/head). The authors suggest that this could be related to the constructive role of LST in improving the $\mathrm{Fl}$, apparent digestibility, and efficiency of microbial $\mathrm{N}$ synthesis in cattle [2], which may be one of the motives that boosted poultry growth in our study.

As natural additives in animal feed, several phytohormones found in medicinal plants are proven to promote growth and feed utilization. Suthikrai et al. [21] found LST contains considerable levels of natural phytoestrogens (10.76-14.55pg/g DM) and phytoandrogens $(0.15-0.92 \mathrm{pg} / \mathrm{g} \mathrm{DM})$. Therefore, it is reasonable to hypothesize that the production of growth hormone and insulin-like growth factor-I may be modulated by phytohormones, resulting in the improvement of growth. Phytoandrogens may also enhance protein synthesis by activating the androgen receptor (AR)-mediated anabolism [28]. In addition, combined with the findings of Buszczak et al. [29], it is possible that the growth-promoting effect of LST may be due to modulating related genes expression related to protein synthesis and cell growth. Moreover, LST is excessively rich in alkaloids, flavonoids, terpenoids, phenolic compounds, steroids, phytosterols, saponins, coumarins, tannins, glycosides and anthraquinone [30]. Kikusato et al. [31] found that plant-derived isoquinoline alkaloids supplementation significantly improved the growth performance of chickens under heat stress conditions. Starčević et al. [32] reported that phenolic compounds boosted the growth performance and antioxidant profile of chickens. It has been reported that phytosterols could promote animal production and antioxidant capacity. Thus, these phytochemical constituents may collectively contribute to the growth-promoting property of LST.

Blood variables are commonly reliable indicators of general health profile, as responses to external and internal stressors and stimuli. The study notices LST supplements did not significantly affect the levels of 
blood metabolites (except for TBIL, TG, HDL-C and LDL-C), showing that LST had no detrimental impacts or toxicity in the broilers. In the present study, dietary inclusion of LST reduced the triglyceride and LDL-C and boosted TBIL and HDL-C levels in broiler serum. The findings were consistent with prior studies in rats where the levels of triglyceride and LDL-C were significantly decreased with the methanolic leaves extract of LST $(200,400,800 \mathrm{mg} / \mathrm{kg} \mathrm{BW})$ administration in compared to the control groups [12]. Moreover, Kaewamatawong et al. [33] studied the acute to subchronic toxicity of LST in mice and found that LST significantly decreased the triglyceride values but did not induce any toxicological effects in the acute and subchronic term. The presence of anti-hypertensive compounds such as flavonoids and alkaloids present in LST could be responsible to reduce serum triglycerides and LDL-C and enhancing the HDL-C levels and thus avoiding cardiovascular ailment [24]. Further studies should be conducted to validate the underlined mechanism of LST as an anti-hypertensive agent for drug treatments in poultry. The present study has shown that LST significantly improved the levels of $\mathrm{Ca}$, Fe and $\mathrm{P}$ in broiler serum as a response to $4 \%$ addition of LST in broiler diets. This enhancement could be due to the rich trace element content in LST [11]. In addition, TBIL was significantly reduced only in the LST1 groups and other biochemical parameters were similar among the groups, thus indicating that the LST powder consumption did not have a negative influence on the health status of the animals.

The immunity of poultry is usually affected by the animal's oxidative status (OS) [8]. An excess amount of OS can cause serious damage to DNA, lipids and proteins. The elimination of extra OS depends on the action of several enzymes such as SOD and CAT [10]. Moreover, chicken meat is relatively rich in polyunsaturated fatty acids, making it vulnerable to oxidation $[11,24]$. Therefore, diet-derived antioxidants may be vital in reducing cumulative oxidative damage. Natural antioxidants are broadly found in medicinal herbs. The LST and its extracts have antioxidant property due to its high content of flavonoids and phenolic compounds [19]. It has been reported that the extracts of LST could scavenge the stable radical 1,1-diphenyl-2-pecrylhydrazyl (DPPH) [14], elucidated those polyphenols and ascorbic acid contributed mainly to the total antioxidant activity of rhizomes. Besides, phytochemical constituents in LST such as phytosterols [34] and flavonoids [35], were proven to improve the antioxidant enzyme activity.

In the present study, we measure the levels of antioxidant enzymes to determine the antioxidant status in serum, liver and breast muscle. We found that GSH-Px, SOD and CAT were significantly increased in LST supplemented groups, while the levels of ROS and MDA, as indicators of OS, were significantly reduced. The increased antioxidant enzyme content is parallel with the decreased level of MDA and ROS as response to dietary LST inclusion, indicating that dietary LST supplement can promote the antioxidant capability of birds by enhancing the enzymatic antioxidant scheme. As the improvement of the antioxidant enzyme would exert a positive influence on meat quality [34], so it is logical to speculate that the addition of LST can enhance the meat quality of the chickens involved in this experiment.

Results of the current study indicated that the dietary LST powder addition affected the structure of the intestine in broilers. It is well known that intestinal morphology can objectively reflect gut health, and villus height as well as crypt depth can partially reflect functional status of the intestine [22]. A previous study demonstrated that the villus height was increased and the intestinal function was improved in fish after supplementation of LST extract in diet [20]. Similarly, in this study, a statistical decrease in crypt depth and 
increase in villus height and in the ratio of the two was observed in the intestine of chickens fed LST powder compared with the control groups. The increased villus height and villus height to crypt depth ratio for various gut fractions of chickens fed LST powder were in accordance with appropriate growth performance and increased metabolizability of nutrients. In fact, the increase of villus height (Vh) usually results in higher total luminal villus absorptive area and subsequently lead to higher transport of nutrients at the villus surface [36]. Moreover, the decrease in crypt depth (Cd) usually indicates a slower turnover and healthier condition of the gut, which can reduce maintenance requirement and ultimately be beneficial to promote the growth of the animals [37]. It is possible that the richness of fiber fractions in LST rhizome sustain the intestinal integrity through increasing the $\mathrm{Vh}$ and $\mathrm{Vh} / \mathrm{Cd}$, decreasing the $\mathrm{Cd}$ feature [4].

The gut microbiome is closely related to intestinal health, normal physiological functions and poultry production, and the composition of the microbial community could be altered upon the diet and over time [39]. This study is setup necessarily to evaluate the effect of LST powder as a feed additive on the intestinal flora of Chinese yellow-feathered broilers, which will contribute to developing nutrition intervention for optimal health, growth and productivity in poultry. We found that the dominant phyla in the chicken are Firmicutes, Bacteroidetes, Proteobacteria. Furthermore, the primary genera were Bacteroides, Faecalibacterium, Oscillospira in the current study, which was consistent with earlier works recognizing Bacteroidetes as the fundamental genus in the cecum (about $40 \%$ of sequences) [40].

Dietary LST powder addition also caused a significant alteration of the gut microbiota diversity in broilers. According to the a-diversity results, the $1 \%$ supplementation LST powder significantly increased the abundance of caecal microbiota (PD whole tree index, Chao1 index, observed species and unique OTUs) compared with the other groups, while $2 \%$ and $4 \%$ supplementation LST powder decreased the abundance (chao1 index and observed species) of caecal micorbiota. In addition, regarding the $\beta$-diversity analysis, a clear separation of microbial community due to dietary LST powder inclusion was observed, which means microbial community of each group were significantly different. Elevated levels of diversity mostly contribute to maintaining the stability of intestinal microbiota after exposing to environmental stress, as well as resistance against potential invading pathogens [41]. For these reasons, it is considered that a reduction of a and $\beta$ diversity should be negative. This suggests that the structure of intestinal flora in the LST1 groups was improved and, thus, better intestinal health. However, LST2 and LST4 groups got a lower diversity, and this may be resulted from the anti-bacterial activity of higher LST powder addition [13, 14].

LEfSe analysis revealed that the relative abundance of Anaerofilum was significantly increased in the LST1 groups compared with other groups, which has been proposed to boost the antioxidant capability and absorption of energy as previously reported by Guo et al. [42]. Interestingly, Odoribacter [43] and Sutterella [44], known as SCFA-producing bacteria, were also overrepresented in the LST1 groups. SCFAs, as the main energy source of colorectal cells, play an essential role in regulating the absorption of several nutrients, digestive and hormonal secretions in the intestine, and participating in energy metabolism widely [43, 44]. In addition, Bacillus has been widely proven to promote growth performance $[43,45]$. In this study, we found the enrichment of Bacillus in the LST1 groups, while the best growth performance was found in the LST1 groups. Therefore, the increase of relative abundance in Anaerofilum, Odoribacter, Sutterella and Bacillus in LST1 groups in the present study, suggests the efficacy of $1 \%$ LST powder in promoting beneficial bacteria, which 
would eventually contribute to improved performance and health. These findings suggest that there is close association between intestinal flora and physiological performance.

However, no reports have verified the associations of growth performance, serum metabolites, antioxidant status and intestinal morphology with changed gut microbiome structure in Chinese yellow-feathered broilers fed with LST powder. By using Spearman's correlation analysis, we revealed that the profiles of taxonomic composition at the genus level in cecal microbiota were significantly associated with growth performance, serum metabolites, antioxidant status and intestinal morphology. Consistently, the relative abundance of Odoribacter and Bacillus was positively correlated with growth performance, which showed that $1 \%$ addition level of LST powder could improve ADG and decrease FCR potentially by increasing the abundance of Odoribacter and Bacillus as detected at day 42. In the present study, we also demonstrated that Bacillus, which has been reported to improve intestinal morphology $[46,47]$, was positively related to the $\mathrm{Vh}$ and $\mathrm{Vh} / \mathrm{Cd}$ of duodenum. Furthermore, the abundance of Bacillus was closely correlated to the concentrations of CAT, SOD and GSH-Px, and many studies have reported Bacillus could participate in boosting of antioxidant scheme $[48,49]$. Actually, intestinal flora can regulate the host well-being, immunity and disease by determining the biological value of the diet [48]. Here, we found that serum metabolites including triglyceride, TBIL, Ca, Mg and LDL-C affected by dietary treatment of LST powder, were faithfully connected with the dynamic fluctuations of broiler gut microbiome structure.

Overall, the gut microbiota is reshaped by LST supplements and further contributes to improvement of growth performance, intestinal morphology, antioxidant capacity, $\mathrm{Ca}$ and $\mathrm{Mg}$, and reduction of triglyceride, LDL-C and TBIL.

\section{Conclusion}

Our results indicate that the beneficial effects of LST powder especially $1 \%$ in the improvement of growth performance, intestinal morphology, antioxidant capacity, $\mathrm{Ca}$ and $\mathrm{Mg}$, and reduction of triglyceride, LDL-C and TBIL could be partially attributed to modulating gut microbiota structure and diversity in Chinese yellowfeathered broilers. Of note, during the antibiotic-free era, LST could have great uses in broiler feeding when the importance of nutrition, feed processing, management, biosafety strategies is highly recognized.

\section{Abbreviations}

LST: Lasia spinosa Thw.; LST0 = control diet; LST1 $=1 \%$ supplementation level of Lasia spinosa Thw. powder; LST2 $=2 \%$ supplementation level of Lasia spinosa Thw. powder; LST4 $=4 \%$ supplementation level of Lasia spinosa Thw. powder; BW: body weight; ADFI: average daily feed intake; ADG: average daily gain; FCR: feed conversion ratio; Vh: villus height; Cd: crypt depth; Vh/Cd: villus height to crypt depth ratio; ROS: reactive oxygen species; MDA: malondialdehyde; SOD: superoxide dismutase; CAT: catalase; GSH-Px: glutathione peroxidase; ALT: alanine aminotransferase; AST: aspartate aminotransferase; DBIL: direct bilirubin; TBIL: total bilirubin; HDL-C: high-density lipoproteincholesterol; LDL-C: low-density lipoproteincholesterol; RF: rheumatoid factors; PCA: principle component analysis; LDA: Linear discriminant analysis; LEfSe: Linear discriminant analysis effect size 


\section{Declarations}

\section{Acknowledgements}

The authors thank the Liping Pu and Sile Wu for assistance in editing pictures.

\section{Authors' contributions}

Pre-experiment guidance : YYL, WS, KS and TT. Conceived and designed the experiment: YXH and LZ; Conducted the experiments: YXH, LZ, KT, HDS and YFT; Performed data analysis: YXH and LZ; Writingoriginal draft: $Y X H, X T L$, SAA and AAH; Drafted and revised the manuscript: YQL, YXH and LZ; All authors read and approved the final manuscript.

\section{Funding}

This study was jointly supported by the National Natural Science Foundation of China

(No.31960157), the Guangxi Pioneer of Animal Science and Technology (No. 202109-02) and the Guangxi Special Fund for Invited Expert.

\section{Availability of data and materials}

The datasets used and analyzed during the current study are available from the corresponding author on reasonable request.

Ethics approval and consent to participate

The study procedures were adopted after approval by Guangxi University (GXU2018-003)

\section{Consent for publication}

Not applicable.

\section{Competing interests}

The authors declare that they have no competing interests.

\section{Author details}

${ }^{1}$ State Key Laboratory for Conservation and Utilization of Subtropical Agro-bioresources, College of Animal Science and Technology, Guangxi University, Nanning 530004, Guangxi, China

${ }^{2}$ Guangxi Fufeng Agriculture and Animal Husbandry Co Ltd, Nanning 530001, China 
${ }^{3}$ Faculty of Agriculture, Zagazig University, Zagazig, 44511, Egypt

${ }^{4}$ Guangxi Veterinary Research Institute, Nanning 530001, Guangxi, China

${ }^{5}$ Research and Development Center for Livestock Production Technology, Faculty of Veterinary Science, Chulalongkorn University, Bangkok 10330, Thailand

${ }^{6}$ Department of Obstetrics, Gynecology and Reproduction, Faculty of Veterinary Science,

Chulalongkorn University, Bangkok 10330, Thailand

\section{References}

1. Dibner JJ, Richards JD. Antibiotic growth promoters in agriculture: history and mode of action. Poul Sci J. 2005;84:634-43.

2. Phesatcha K, Foiklang S, Wanapat M. Effect of Lasia spinosa supplementation on nutrients digestibility and microbial protein synthesis in Thai native beef cattle. J Agric Res Ext. 2018;35(2 Suppl. 2):160-8.

3. Food and Agriculture Organization of the United Nations (FAO). In: Organisation for Economic CoOperation and Development (OECD) 2016.

4. Yang X, Liu Y, Yan F, Yang C, Yang X. Effects of encapsulated organic acids and essential oils on intestinal barrier, microbial count, and bacterial metabolites in broiler chickens. Poul Sci J. 2019;98:2858-65.

5. Castanon JIR. History of the Use of Antibiotic as Growth Promoters in European Poultry Feeds. Poul Sci J. 2007;86:2466-71.

6. Agostini P, Sola-Oriol D, Nofrarías M, Barroeta A, Gasa J, Manzanilla E. Role of in-feed clove supplementation on growth performance, intestinal microbiology, and morphology in broiler chicken. Lives Sci. 2012;147(1-3):113-8.

7. Abd El-Hack ME, Alagawany M, Abdel-Moneim AME, Mohammed NG, Khafaga AF, Bin-Jumah M, et al. Cinnamon (Cinnamomum zeylanicum) Oil as a Potential Alternative to Antibiotics in Poultry. Antibiotics. 2020a;9:210.

8. Abd El-Hack ME, Abdelnour SA, Taha AE, Khafaga AF, Arif M, Ayasan T, et al. Herbs as thermoregulatory agents in poultry: An overview. Sci Total Env. 2020b;703:134399.

9. Shefana AG, Ekanayake S. Some nutritional aspects of Lasia spinosa (kohila). Vidyodaya J Sci. 2009;14:59-64.

10. Sharma P, Jha AB, Dubey RS, Pessarakli M. Reactive Oxygen Species, Oxidative Damage, and Antioxidative Defense Mechanism in Plants under Stressful Conditions. J Bot. 2012; 1-26.

11. Brahma J, Chakravarty S, Rethy P. Qualitative estimation of the presence of bioactive and nutritional compound in Lasia spinosa: An important vegetable plant used by the bodos of Kokrajhar district. Inter J Chem Tech Res. 2014;6:1405-12. 
12. Mahmood SB, Atif M, Rashid S, Ahmed MI, Rahman SA. Evaluation of Antihyperlipidemic Activity of Methanolic Leaves Extract of Lasia spinosa and Its Role in Prevention of Hyperlipidemia Induced Pancreatitis in Rats. Int J Pharmac Scie Res. 2015;6:1502-8.

13. Hasan MN, Munshi M, Rahman MH, Alam SN, Hirashima A. Evaluation of Antihyperglycemic Activity of Lasia spinosa Leaf Extracts in Swiss Albino Mice. World J Pharm Pharmac Sci. 2014;10:118-24.

14. Durajan G, Mostafizur RM, Abdul MM, Saiful IM, Mukhtar A. Antioxidant Property, Cytotoxicity and Antimicrobial Activity of Lasia spinosa Leaves. Nepal J Sci Technol. 2013; 13(2).

15. Goshwami D. Rahman MM, AMuhit M, Islam MS. Antinociceptive activity of leaves of Lasia spinosa. Arch Appli Sci Res. 2012;6:2431-4.

16. Deb D. Dev S, Das AK, Khanam D, Basher SK. Antinociceptive. Anti-inflammatory and Anti-diarrheal Activities of the Hydroalcoholic Extract of Lasia spinosa Linn. (Araceae) Roots. Latin Amer J Pharm. 2010;29:1269-76.

17. Jintana R, Suthikrai W, Sophon S, Hengtrakulsin R, Usawang S, Kamonpatana M, et al. Effects of Lasia spinosa Thw. and season on plasma leptin and glucose of weaned female murrah $\times$ swamp buffalo calves. Buffalo Bull. 2013;32:947-50.

18. Muthukrishnan S, Sivakkumar T. Physicochemical Evaluation. Preliminary Phytochemical Investigation, Fluorescence and TLC Analysis of Leaves of Schleichera Oleosa (Lour.) Oken. Indian J Pharmac Sci. 2018;80:525-32.

19. Rahman A, Siddiqui SA, Oke-Altuntas F, Okay S, Gül F, Demirtas I. Phenolic profile, essential oil composition and bioactivity of Lasia spinosa (L.) thwaites. Bra Arch Biol Technol. 2019; 62.

20. Munglue P, Rattana K, Sangchanjiradet S, Dasri K. Effect of Dietary Lasia (Lasia spinosa (L.) Thwaites) Extract on Growth Performance and Intestinal Histology in Hybrid Catfish (Clarias macrocephalus $\mathrm{x}$ Clarias gariepinus). Chiang Mai Uni J Nat Sci. 2019;18:226-49.

21. Suthikrai W, Jintana R, Sophon S, Hengtakulsin R, Kamonpatana M. Effects of Lasia spinosa Thw. on growth rate and reproductive hormone of weaned Swamp buffalo and Murrah X Swamp buffalo calves. Ital J Anim Sci. 2007;6:532-5.

22. Laudadio V, Passantino L, Perillo A, Lopresti G, Passantino A, Khan RU, et al. Productive performance and histological features of intestinal mucosa of broiler chickens fed different dietary protein levels. Poul Sci J. 2012;91:265-70.

23. Qaisrani SN, Moquet PA, Van Krimpen MM, Kwakkel RP, Verstegen MA, Hendriks WH. (2014). Protein source and dietary structure influence growth performance, gut morphology, and hindgut fermentation characteristics in broilers. Poul Sci J. 2014; 93: 3053.

24. Alagawany M, El-Saadony MT, Elnesr SS, Farahat M, Attia G, Madkour M, et al. Use of lemongrass essential oil as a feed additive in quail's nutrition: its effect on growth, carcass, blood biochemistry, antioxidant and immunological indices, digestive enzymes and intestinal microbiota. Poul Sci J. 2021;100:101172.

25. Alagawany M, Elnesr SS, Farag MR. Use of liquorice (Glycyrrhiza glabra) in poultry nutrition: Global impacts on performance, carcass and meat quality. World's Poul Sci J. 2019;75:293-304. 
26. Ismail IE, Abdelnour SA, Shehata SA, Abd El-Hack ME, El-Edel MA, Taha AE, et al. Effect of Dietary Boswellia serrata Resin on Growth Performance, Blood Biochemistry, and Cecal Microbiota of Growing Rabbits. Front Vet Sci. 2019. https://doi.org/10.3389/fvets.2019.00471.

27. Mahfuz S, Shang Q, Piao X. Phenolic compounds as natural feed additives in poultry and swine diets: a review. J Anim Sci Biotechnol. 2021;12(1). https://doi.org/10.1186/s40104-021-00565-3.

28. Ong VY, Tan BK. Novel phytoandrogens and lipidic augmenters from Eucommia ulmoides. BMC Complem Altern Med. 2007;7:3.

29. Buszczak M, Signer RA, Morrison SJ. Cellular differences in protein synthesis regulate tissue homeostasis. Cell. 2014;159:242-51.

30. Van NH, Van Minh C, De Leo M, Siciliano T, Braca A. (. Secondary metabolites from Lasia spinosa (L.) Thw.(Araceae). Biochem Syst Ecol. 2006;12:882-4.

31. Kikusato M, Xue G, Pastor A, Niewold TA, Toyomizu M. Effects of plant-derived isoquinoline alkaloids on growth performance and intestinal function of broiler chickens under heat stress. Poul Sci J. 2021;100:957-63.

32. Starčević K, Krstulović L, Brozić D, Maurić M, Stojević Z, Mikulec Ž, et al. Production performance, meat composition and oxidative susceptibility in broiler chicken fed with different phenolic compounds. J Sci Food Agri. 2015;95(6):1172-8.

33. Kaewamatawong T, Suthikrai W, Bintvihok A, Banlunara W. Acute to subchronic toxicity and reproductive effects of aqueous ethanolic extract of rhizomes of Iasia spinosa thw. in male rats. Thai J Vet Med. 2013;43:69-74.

34. Zhao YR, Chen YP, Cheng YF, Qu HM, Li J, Wen C, et al. Effects of dietary phytosterols on growth performance, antioxidant status, and meat quality in Partridge Shank chickens. Poult Sci. 2019;98:371521.

35. Cheng YF, Hu Q, Wang CM, Xia SS, Liu CX, Wen C, et al. Effects of common and emulsified phytosterols on growth performance, serum biochemical indexes and nutrient digestibility in finishing pigs. $J$ Chinese Cereals Oils Associ. 2017;32:98-102.

36. Tufarelli V, Desantis S, Zizza S, Laudadio V. Performance, gut morphology and carcass characteristics of fattening rabbits as affected by particle size of pelleted diets. Archiv Anim Nut. 2010;64:373-82.

37. Van Nevel CJ, Decuypere JA, Dierick NA, Molly K. Incorporation of galactomannans in the diet of newly weaned piglets: effect on bacteriological and some morphological characteristics of the small intestine. Archiv Anim Nut. 2005;59(2):123-38.

38. Jazi V, Ashayerizadeh A, Toghyani M, Shabani A, Tellez G, Toghyani M. Fermented soybean meal exhibits probiotic properties when included in Japanese quail diet in replacement of soybean meal. Poult Sci. 2018;97(6):2113-22. https://doi.org/10.3382/ps/pey071.

39. Tilocca B, Burbach K, Heyer CM, Hoelzle LE, Mosenthin R, Stefanski V, et al. Dietary changes in nutritional studies shape the structural and functional composition of the pigs' fecal microbiome-from days to weeks. Microbiome. 2017;5:1-15.

40. Xiao Y, Xiang Y, Zhou W, Chen J, Li K, Yang H. Microbial community mapping in intestinal tract of broiler chicken. Poul Sci. 2017;96:1387-93. 
41. Oakley BB, Lillehoj HS, Kogut MH, Kim WK, Maurer JJ, Pedroso A, et al. The chicken gastrointestinal microbiome. FEMS Mlcrobiol lett. 2014;360:100-12.

42. Guo Y, Liu CQ, Shan CX, Chen Y, Li HH, Huang ZP, et al. Gut microbiota after Rouxeny gastric bypass and sleeve gastrectomy in a diabetic rat model: Increased diversity and associations of discriminant genera with metabolic changes. Diabetes Metab Res Rev. 2017;33(3):e2857.

43. Wang F, Yu T, Huang G, Cai D, Liang X, Su H, et al. Gut microbiota community and its assembly associated with age and diet in Chinese centenarians. J Microbiol Biotechnol. 2015;25:1195-204.

44. Allaire JM, Crowley SM, Law HT, Chang SY, Ko HJ, Vallance BA. The intestinal epithelium: central coordinator of mucosal immunity. Trends in immunol. 2018;39:677-96.

45. Zhu C, Gong L, Huang K, Li F, Tong D, Zhang H. Effect of heat-inactivated compound probiotics on growth performance, plasma biochemical indices, and cecal microbiome in Yellow-Feathered broilers. Front Microbiol. 2020; 11.

46. Al-Fataftah AR, Abdelqader A. Effects of dietary Bacillus subtilis on heat-stressed broilers performance, intestinal morphology and microflora composition. Anim Feed SciTechnol. 2014;198:279-85.

47. Lei X, Piao X, Ru Y, Zhang H, Péron A, Zhang H. Effect of Bacillus amyloliquefaciens-based direct-fed microbial on performance, nutrient utilization, intestinal morphology and cecal microflora in broiler chickens. Asian- Asian Australas J Anim Sci. 2015;28:239.

48. Rowland I, Gibson G, Heinken A, Scott K, Swann J, Thiele I, et al. Gut microbiota functions: metabolism of nutrients and other food components. Eur J Nutr. 2018;57:1-24.

49. National Research Council. Nutrient requirements of poultry: 1994. National Academies Press; 1994.

\section{Tables}

Table 1 Effect of dietary LST powder supplementation on growth performance of broiler chickens. 
Treatment $^{2}$

Item $^{1} \quad$ LST0 $\quad$ LST1 $\quad$ LST2 $\quad$ LST4
SEM $^{4}$

P-value ${ }^{3}$

ANOVA Linear Quadratic

\section{Body wight (BW; g)}

$\begin{array}{lllllllll}\text { At one d } & 42.57 & 42.35 & 42.62 & 42.55 & 0.06 & 0.3275 & 0.6381 & 0.5199\end{array}$

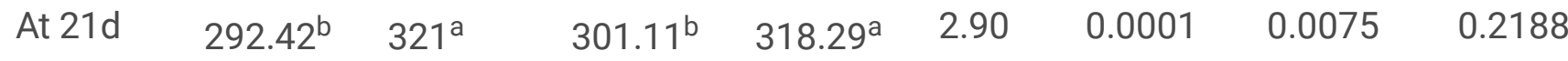

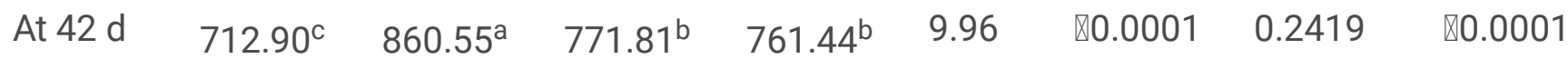

\section{Average daily gain (ADG; g)}

\begin{tabular}{|c|c|c|c|c|c|c|c|c|}
\hline $1-21 d$ & $11.90^{\mathrm{b}}$ & $13.27^{\mathrm{a}}$ & $12.31^{b}$ & $13.13^{\mathrm{a}}$ & 0.88 & 0.0001 & 0.0079 & 0.2150 \\
\hline & $20.02^{c}$ & $25.69^{a}$ & $22.41^{b}$ & $21.10^{b c}$ & 0.42 & 0.0001 & 0.9853 & .0001 \\
\hline $1-42 d$ & $15.96^{c}$ & $19.48^{\mathrm{a}}$ & $17.36^{\mathrm{b}}$ & $17.12^{b}$ & 0.24 & $\llbracket 0.0001$ & 0.2444 & $\otimes 0.0001$ \\
\hline
\end{tabular}

\section{Average Daily Feed Intake (ADFl; g)}

$\begin{array}{lllllllll}1-21 d & 24.15 & 24.88 & 24.67 & 25.37 & 1.11 & 0.0916 & 0.0261 & 0.9617\end{array}$

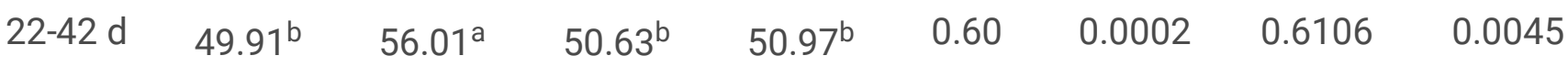

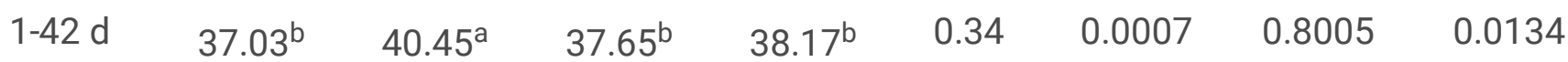

\section{Feed Conversion Rate (FCR; $g$ BW/g feed)}

\begin{tabular}{|c|c|c|c|c|c|c|c|c|}
\hline $1-21 d$ & $2.03^{a}$ & $1.88^{\mathrm{c}}$ & $2.00^{\mathrm{a}}$ & $1.93^{b}$ & 0.01 & $\llbracket 0.0001$ & 0.0371 & 0.0239 \\
\hline $22-42$ & $2.51^{a}$ & $2.18^{b}$ & $2.26^{\mathrm{b}}$ & $2.42^{a}$ & 0.03 & $\llbracket 0.0001$ & 0.3850 & $\varangle 0.0001$ \\
\hline $1-42 \mathrm{~d}$ & $2.27^{a}$ & $2.03^{c}$ & $2.14^{\mathrm{b}}$ & $2.18^{b}$ & 0.18 & 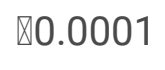 & 0.0902 & $\llbracket 0.0001$ \\
\hline
\end{tabular}

\section{Mortality (\%)}

$\begin{array}{lllllllll}1-21 \mathrm{~d} & 0 & 2.00 & 1.00 & 2.00 & 0.53 & 0.4994 & 0.3018 & 0.6422 \\ 22-42 \mathrm{~d} & 0 & 0 & 0 & 0 & 0 & - & - & - \\ 1-42 \mathrm{~d} & 0 & 1 & 0.5 & 1 & 0.26 & 0.4994 & 0.3018 & 0.6422\end{array}$

${ }^{1} B W$ : body weight; $A D G$ : average daily gain; $A D F l$ : average daily feed intake; $F C R$ : feed conversion ratio.

2LST0: control diet; LST1: 1\% supplementation level of Lasia spinosa Thw. powder; LST2: $2 \%$ supplementation level of Lasia spinosa Thw. powder; LST4: 4\% supplementation level of Lasia spinosa Thw. powder.

${ }^{3}$ Mean values within a row with different letters differ at $\mathrm{P}<0.05$. 
${ }^{4}$ SEM: standard error of the mean.

Table 2 Effect of dietary LST powder supplementation on blood metabolites and mineral profile of broiler chickens $(n=10)$.

\begin{tabular}{|c|c|c|c|c|c|c|c|c|}
\hline \multirow[b]{2}{*}{ Item 1} & \multicolumn{5}{|c|}{ Treatment $^{2}$} & \multicolumn{3}{|l|}{$P$-value ${ }^{3}$} \\
\hline & LSTO & LST1 & LST2 & LST4 & SEM $^{4}$ & ANOVA & Linear & Quadratic \\
\hline \multicolumn{9}{|l|}{ Blood Metabolites } \\
\hline $\mathrm{ALT}, \mathrm{U} / \mathrm{L}$ & 2 & 2.1 & 2.1 & 2 & 0.08 & 0.9463 & 1.0000 & 0.5483 \\
\hline AST, U/L & 212.2 & 216.1 & 222 & 206.2 & 3.13 & 0.3480 & 0.6670 & 0.1230 \\
\hline Total protein, g/L & 37.83 & 39.22 & 38.27 & 39.28 & 0.64 & 0.8305 & 0.5694 & 0.8867 \\
\hline Albumin, g/L & 15.23 & 15.9 & 15.67 & 16.11 & 0.20 & 0.4480 & 0.1825 & 0.7734 \\
\hline DBIL, $\mu \mathrm{mol} / \mathrm{L}$ & 3.76 & 3.63 & 3.79 & 3.79 & 0.05 & 0.5828 & 0.5559 & 0.4938 \\
\hline TBIL, $\mu \mathrm{mol} / \mathrm{L}$ & $22.87^{a}$ & $14.68^{b}$ & $22.69^{a}$ & $21.97^{a}$ & 0.71 & $\begin{array}{l}\square \\
0.0001\end{array}$ & 0.2211 & 0.0004 \\
\hline Urea, mmol/L & 0.85 & 0.90 & 0.82 & 0.85 & 0.02 & 0.3570 & 0.5288 & 0.7677 \\
\hline Uric Acid, $\mu \mathrm{mol} / \mathrm{L}$ & 106 & 114.9 & 105.3 & 112.8 & 6.15 & 0.9332 & 0.8506 & 0.9564 \\
\hline $\begin{array}{l}\text { Triglyceride, } \\
\mathrm{mmol} / \mathrm{L}\end{array}$ & $0.65^{a}$ & $0.467^{b}$ & $0.523^{b}$ & $0.512^{b}$ & 0.02 & 0.0061 & 0.0323 & 0.0208 \\
\hline Cholesterol, mmol/L & 4.20 & 4.37 & 4.36 & 4.13 & 0.08 & 0.6395 & 0.7759 & 0.2112 \\
\hline HDL_C, mmol/L & $1.42^{\mathrm{b}}$ & $1.66^{\mathrm{a}}$ & $1.6^{\mathrm{a}}$ & $1.625^{\mathrm{a}}$ & 0.02 & $\begin{array}{l}\square \\
0.0001\end{array}$ & 0.0003 & 0.0015 \\
\hline LDL_C, mmol/L & $1.60^{\mathrm{a}}$ & $0.84^{d}$ & $1.42^{\mathrm{b}}$ & $1.13^{\mathrm{c}}$ & 0.06 & $\begin{array}{l}\square \\
0.0001\end{array}$ & 0.0049 & 0.0007 \\
\hline Glucose, $\mathrm{mmol} / \mathrm{L}$ & 10.56 & 10.31 & 10.11 & 10.47 & 0.18 & 0.8371 & 0.7789 & 0.4205 \\
\hline $\mathrm{RF}, \mathrm{IU} / \mathrm{mL}$ & 3.45 & 3.42 & 3.79 & 3.55 & 0.09 & 0.4480 & 0.4012 & 0.5553 \\
\hline \multicolumn{9}{|l|}{ Mineral Profile } \\
\hline Calcium, mmol/L & $7.07^{d}$ & $7.80^{\mathrm{a}}$ & $7.38^{\mathrm{c}}$ & $7.61^{b}$ & 0.05 & $\begin{array}{l}\square \\
0.0001\end{array}$ & $\begin{array}{l}\square \\
0.0001\end{array}$ & $\varangle 0.0001$ \\
\hline Iron, $\mu \mathrm{mol} / \mathrm{L}$ & $15.91^{c}$ & $17.9^{\mathrm{b}}$ & $18.88^{\mathrm{ab}}$ & $19.63^{a}$ & 0.35 & 0.0002 & $\begin{array}{l}0 \\
0.0001\end{array}$ & 0.2684 \\
\hline $\begin{array}{l}\text { Magnesium, } \\
\mathrm{mmol} / \mathrm{L}\end{array}$ & $0.87^{b}$ & $0.85^{\mathrm{b}}$ & $0.91^{\mathrm{b}}$ & $0.98^{a}$ & 0.01 & 0.0025 & 0.0008 & 0.0660 \\
\hline $\begin{array}{l}\text { Phosphorus, } \\
\mathrm{mmol} / \mathrm{L}\end{array}$ & $2.10^{c}$ & $2.31^{\mathrm{b}}$ & $2.39^{b}$ & $2.56^{\mathrm{a}}$ & 0.03 & $\begin{array}{l}\square \\
0.0001\end{array}$ & $\stackrel{\square}{0.0001}$ & 0.6816 \\
\hline
\end{tabular}


${ }^{1} A L T$ : alanine aminotransferase; $A S T$ : aspartate aminotransferase; $D B I L$ : direct bilirubin; $T B I L$ : total bilirubin; $H D L-C$. high-density lipoprotein cholesterol; $L D L-C$. low-density lipoprotein cholesterol and $R F$ : rheumatoid factors.

2LST: Lasia spinosa Thw.; LST0: control diet; LST1: 1\% supplementation level of Lasia spinosa Thw. powder; LST2: $2 \%$ supplementation level of Lasia spinosa Thw. powder; LST4: $4 \%$ supplementation level of Lasia spinosa Thw. powder.

${ }^{3}$ Mean values within a row with different letters differ at $\mathrm{P}<0.05$.

${ }^{4}$ SEM: standard error of the mean

Table 3 Effect of dietary LST powder supplementation on serum, liver, and muscle antioxidant status of broiler chickens $(n=6)$. 


\begin{tabular}{|c|c|c|c|c|c|c|c|c|}
\hline \multicolumn{4}{|c|}{ Treatment $^{2}$} & \multicolumn{5}{|c|}{$P$-value ${ }^{3}$} \\
\hline Item ${ }^{1}$ & LSTO & LST1 & LST2 & LST4 & SEM $^{4}$ & ANOVA & Linear & Quadratic \\
\hline \multicolumn{9}{|l|}{ Serum } \\
\hline ROS, IU/mL & $468.28^{a}$ & $449.93^{\mathrm{a}}$ & $347.53^{b}$ & $363.49^{b}$ & 16.91 & 0.0111 & 0.0030 & 0.5418 \\
\hline $\begin{array}{l}\mathrm{MDA} \\
\mathrm{nmol} / \mathrm{mL}\end{array}$ & $8.13^{\mathrm{a}}$ & $7.52^{\mathrm{ab}}$ & $6.42^{b}$ & $6.95^{b}$ & 0.22 & 0.0271 & 0.0127 & 0.1466 \\
\hline SOD, ng/mL & $3.52^{b}$ & $4.52^{\mathrm{a}}$ & $4.60^{\mathrm{a}}$ & $5.35^{\mathrm{a}}$ & 0.21 & 0.0097 & 0.0014 & 0.7311 \\
\hline CAT, pg/mL & $354.02^{c}$ & $448.06^{b}$ & $511.58^{a}$ & $495.54^{\mathrm{ab}}$ & 15.07 & 0.0001 & $\begin{array}{l}\square \\
0.0001\end{array}$ & 0.0043 \\
\hline $\begin{array}{l}\text { GSH-Px, } \\
\text { ng/mL }\end{array}$ & $149.52^{b}$ & $167.36^{\mathrm{ab}}$ & $183.40^{\mathrm{a}}$ & $185.50^{\mathrm{a}}$ & 4.27 & 0.0027 & 0.0004 & 0.2390 \\
\hline \multicolumn{9}{|l|}{ Liver } \\
\hline $\begin{array}{l}\text { ROS, } \\
\text { IU/mg.pro }\end{array}$ & $787.18^{a}$ & $618.71^{b}$ & $601.94^{b}$ & $440.50^{c}$ & 34.03 & 0.0007 & $\stackrel{\square}{0.0001}$ & 0.9426 \\
\hline $\begin{array}{l}\text { MDA, } \\
\text { nmol/mg.pro }\end{array}$ & $14.47^{a}$ & $11.71^{b}$ & $10.96^{b c}$ & $9.17^{c}$ & 0.51 & 0.0002 & $\begin{array}{l}\square \\
0.0001\end{array}$ & 0.4784 \\
\hline $\begin{array}{l}\text { SOD, } \\
\text { ng/mg.pro }\end{array}$ & 14.71 & 14.37 & 13.46 & 14.36 & 0.64 & 0.9245 & 0.7483 & 0.6539 \\
\hline $\begin{array}{l}\text { CAT, } \\
\text { pg/mg.pro }\end{array}$ & $844.19^{b}$ & $1214.18^{a}$ & $1275.68^{a}$ & $1351.24^{\mathrm{a}}$ & 52.36 & 0.0003 & $\begin{array}{l}\square \\
0.0001\end{array}$ & 0.0506 \\
\hline $\begin{array}{l}\text { GSH-Px, } \\
\text { ng/mg.pro }\end{array}$ & 361.38 & 387.06 & 417.96 & 425.84 & 11.61 & 0.1801 & 0.0345 & 0.6914 \\
\hline \multicolumn{9}{|l|}{$\begin{array}{l}\text { Breast } \\
\text { muscle }\end{array}$} \\
\hline $\begin{array}{l}\text { ROS, } \\
\text { IU/mg.pro }\end{array}$ & $798.37^{a}$ & $663.31^{\mathrm{ab}}$ & $535.79^{b}$ & $638.09^{b}$ & 30.22 & 0.0114 & 0.0124 & 0.0263 \\
\hline $\begin{array}{l}\text { MDA, } \\
\text { nmol/mg.pro }\end{array}$ & $14.1^{\mathrm{a}}$ & $12.64^{\mathrm{ab}}$ & $9.98^{c}$ & $11.04^{b c}$ & 0.49 & 0.0081 & 0.0033 & 0.1273 \\
\hline $\begin{array}{l}\text { SOD, } \\
\text { ng/mg.pro }\end{array}$ & $10.83^{b}$ & $13.56^{\mathrm{a}}$ & $15.23^{a}$ & $13.18^{a}$ & 0.49 & 0.0074 & 0.0224 & 0.0064 \\
\hline $\begin{array}{l}\text { CAT, } \\
\text { pg/mg.pro }\end{array}$ & $726.99^{b}$ & $1049.43^{a}$ & $1167.52^{\mathrm{a}}$ & $1164.84^{a}$ & 50.98 & 0.0012 & 0.0003 & 0.0403 \\
\hline $\begin{array}{l}\text { GSH-Px, } \\
\text { ng/mg.pro }\end{array}$ & $304.27^{b}$ & $398.56^{a}$ & $390.45^{a}$ & $380.55^{a}$ & 12.05 & 0.0103 & 0.0205 & 0.0152 \\
\hline
\end{tabular}

${ }^{1}$ ROS. reactive oxygen species; $M D A$ : malondialdehyde; SOD. superoxide dismutase; $C A T$ : catalase; $G S H$ $P X$. glutathione peroxidase. 
2LST: Lasia spinosa Thw.; LST0: control diet; LST1: 1\% supplementation level of Lasia spinosa Thw. powder; LST2: $2 \%$ supplementation level of Lasia spinosa Thw. powder; LST4: $4 \%$ supplementation level of Lasia spinosa Thw. powder.

${ }^{3}$ Mean values within a row with different letters differ at $\mathrm{P}<0.05$.

${ }^{4}$ SEM: standard error of the mean.

Table 4 Effect of dietary LST powder supplementation on the morphology of the small intestine and cecum of yellow-feathered broilers $(n=10)$.

\begin{tabular}{|c|c|c|c|c|c|c|c|c|}
\hline \multirow[t]{2}{*}{ Item $^{1}$} & \multicolumn{5}{|c|}{ Treatment $^{2}$} & \multicolumn{3}{|c|}{$P$-value ${ }^{3}$} \\
\hline & LSTO & LST1 & LST2 & LST4 & SEM $^{4}$ & ANOVA & Linear & Quadratic \\
\hline \multicolumn{9}{|c|}{ Duodenum } \\
\hline$V h, \mu \mathrm{m}$ & $1173.62^{b}$ & $1528.74^{a}$ & $1503.23^{\mathrm{a}}$ & $1524.74^{\mathrm{a}}$ & 36.69 & 0.0001 & 0.0003 & 0.0065 \\
\hline $\mathrm{Cd}, \mu \mathrm{m}$ & $236.77^{a}$ & $180.25^{b}$ & $185.67^{b}$ & $224.09^{a b}$ & 8.71 & 0.0446 & 0.6560 & 0.0059 \\
\hline $\mathrm{Vh} / \mathrm{Cd}$ & $5.09^{b}$ & $9.06^{\mathrm{a}}$ & $8.56^{a}$ & $7.35^{a}$ & 0.42 & 0.0016 & 0.0546 & 0.0008 \\
\hline \multicolumn{9}{|c|}{ Jejunum } \\
\hline$V h, \mu \mathrm{m}$ & $1020.47^{c}$ & $1263.61^{b}$ & $1381.79^{b}$ & $1536.02^{\mathrm{a}}$ & 38.31 & $\begin{array}{l}\square \\
0.0001\end{array}$ & $\begin{array}{l}\square \\
0.0001\end{array}$ & 0.3722 \\
\hline $\mathrm{Cd}, \mu \mathrm{m}$ & $215.42^{\mathrm{a}}$ & $149.14^{b}$ & $188.62^{\mathrm{ab}}$ & $175.26^{\mathrm{ab}}$ & 8.63 & 0.0463 & 0.2683 & 0.1092 \\
\hline $\mathrm{Vh} / \mathrm{Cd}$ & $5.19^{b}$ & $8.56^{a}$ & $7.75^{\mathrm{a}}$ & $9.49^{\mathrm{a}}$ & 0.40 & 0.0002 & 0.0002 & 0.2114 \\
\hline \multicolumn{9}{|l|}{ Ileum } \\
\hline$V h, \mu \mathrm{m}$ & $692.73^{c}$ & $1042.04^{b}$ & $1069.29^{b}$ & $1295.16^{a}$ & 43.66 & $\begin{array}{l}\square \\
0.0001\end{array}$ & $\begin{array}{l}\square \\
0.0001\end{array}$ & 0.2753 \\
\hline $\mathrm{Cd}, \mu \mathrm{m}$ & $144.19^{\mathrm{ab}}$ & $116.15^{b}$ & $133.05^{b}$ & $169.00^{\mathrm{a}}$ & 6.15 & 0.0112 & 0.0650 & 0.0051 \\
\hline $\mathrm{Vh} / \mathrm{Cd}$ & $5.18^{b}$ & $9.27^{a}$ & $8.42^{a}$ & $8.06^{a}$ & 0.41 & 0.0008 & 0.0142 & 0.0023 \\
\hline \multicolumn{9}{|l|}{ Cecum } \\
\hline$V h, \mu \mathrm{m}$ & $757.09^{c}$ & $933.04^{b}$ & $983.65^{\mathrm{ab}}$ & $1126.84^{\mathrm{a}}$ & 32.19 & 0.0001 & $\begin{array}{l}\square \\
0.0001\end{array}$ & 0.7476 \\
\hline $\mathrm{Cd}, \mu \mathrm{m}$ & $158.21^{\mathrm{ab}}$ & $118.48^{b}$ & $151.38^{\mathrm{ab}}$ & $206.27^{a}$ & 11.27 & 0.0446 & 0.0675 & 0.0305 \\
\hline $\mathrm{Vh} / \mathrm{Cd}$ & $5.04^{b}$ & $8.06^{a}$ & $7.36^{\mathrm{a}}$ & $6.51^{\mathrm{ab}}$ & 0.39 & 0.0343 & 0.2575 & 0.0115 \\
\hline
\end{tabular}

${ }^{1}$ Vh: villus height; $C d$ : crypt depth; $V h / C d$ : villus height to crypt depth ratio. 
2LST: Lasia spinosa Thw.; LST0: control diet; LST1:1\% supplementation level of Lasia spinosa Thw. powder; LST2: $2 \%$ supplementation level of Lasia spinosa Thw. powder; LST4: $4 \%$ supplementation level of Lasia spinosa Thw. powder.

${ }^{3}$ Mean values within a row with different letters differ at $\mathrm{P}<0.05$.

${ }^{4}$ SEM: standard error of the mean.

Table 5 The differential genera that were separately correlated with growth performance, blood metabolites, antioxidant status and intestinal morphology in 42-day-old yellow-feathered broilers by using Spearman correlation analyses $(n=5)$. 


\begin{tabular}{|c|c|c|c|}
\hline Genus & Performance $^{1}$ & Correlation coefficient & $P$-value \\
\hline Alistipes & CAT (serum) & -0.491729323 & 0.027658228 \\
\hline Alistipes & Triglyceride & 0.449718312 & 0.046650271 \\
\hline Alistipes & CAT (liver) & -0.469172932 & 0.036896559 \\
\hline Alistipes & Duodenum Vh/Cd & -0.454135338 & 0.044279104 \\
\hline Alistipes & Duodenum Cd & 0.481203008 & 0.031711045 \\
\hline Alistipes & ROS (breast muscle) & 0.461654135 & 0.040457634 \\
\hline Barnesiella & ROS (serum) & 0.491729323 & 0.027658228 \\
\hline Barnesiella & MDA (serum) & 0.552295115 & 0.011566706 \\
\hline Barnesiella & GSH-Px (liver) & -0.568421053 & 0.00892276 \\
\hline Mucispirillum & $\mathrm{Ca}$ & 0.518448942 & 0.019183165 \\
\hline Mucispirillum & Jejunum Cd & -0.562617565 & 0.009810627 \\
\hline Mucispirillum & Jejunum Vh/Cd & 0.516735651 & 0.019655161 \\
\hline Bacillus & CAT (serum) & 0.518689418 & 0.019117646 \\
\hline Bacillus & SOD (serum) & 0.598530933 & 0.005302503 \\
\hline Bacillus & $\mathrm{F}: \mathrm{G}(1-42$ days $)$ & -0.46666282 & 0.038057182 \\
\hline Bacillus & TBIL & -0.580175398 & 0.007324538 \\
\hline Bacillus & LDL-C & -0.596165198 & 0.005533797 \\
\hline Bacillus & $\mathrm{Ca}$ & 0.531902155 & 0.01578292 \\
\hline Bacillus & Duodenum Vh & 0.625895742 & 0.003157542 \\
\hline Bacillus & Duodenum Vh/Cd & 0.499770655 & 0.024846305 \\
\hline Bacillus & SOD (breast muscle) & 0.611706669 & 0.004155213 \\
\hline Bacillus & GSH-Px (breast muscle) & 0.65585045 & 0.001690504 \\
\hline Peptococcus & TBIL & -0.456999133 & 0.042792109 \\
\hline Peptococcus & LDL-C & -0.450993047 & 0.045956176 \\
\hline Peptococcus & $\mathrm{Ca}$ & 0.562620313 & 0.009810191 \\
\hline Anaerofilum & CAT (serum) & -0.481203008 & 0.031711045 \\
\hline Sutterella & $\mathrm{Mg}$ & 0.446032375 & 0.048702635 \\
\hline Gallibacterium & ROS (serum) & -0.498499154 & 0.025275208 \\
\hline Gallibacterium & LDL-C & 0.486560546 & 0.029593969 \\
\hline
\end{tabular}




\begin{tabular}{|llll|} 
Gallibacterium & $\mathrm{Mg}$ & 0.464581954 & 0.039040647 \\
\hline Gallibacterium & Cecum Cd & 0.478801619 & 0.032697613 \\
\hline Odoribacter & ADG (1-42 days) & 0.479699248 & 0.032326069 \\
\hline Odoribacter & $42 \mathrm{~d} \mathrm{BW}$ & 0.479699248 & 0.032326069 \\
\hline
\end{tabular}

${ }^{1} A D G$ : average daily gain; FCR: feed conversion ratio; TBIL: total bilirubin; $L D L-C$. low-density lipoproteincholesterol; $R O S$. reactive oxygen species; $M D A$ : malondialdehyde; SOD: superoxide dismutase; $C A T$ : catalase; GSH-PX: glutathione peroxidase; Vh: villus height; $C d$ : crypt depth; $V h / C d$ : villus height to crypt depth ratio.

\section{Figures}

A

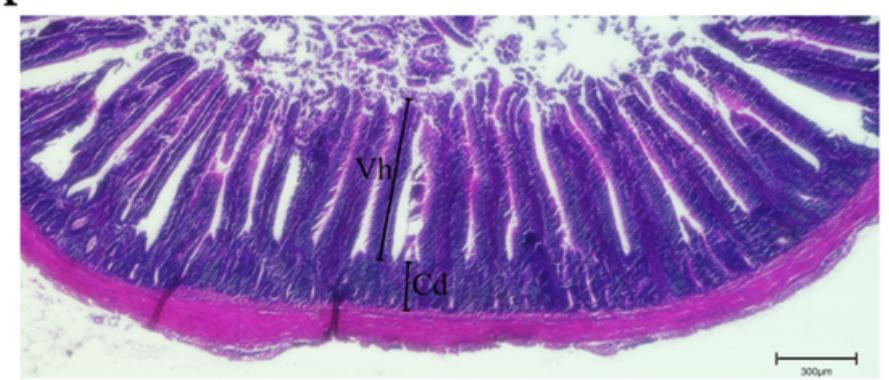

$\mathrm{C}$

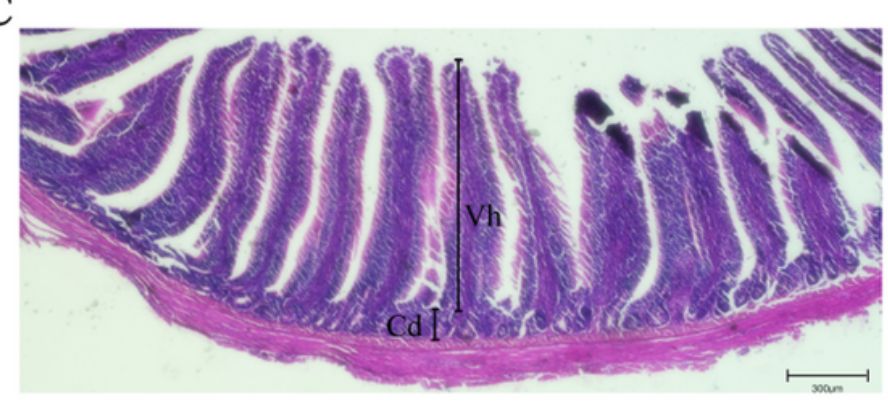

B

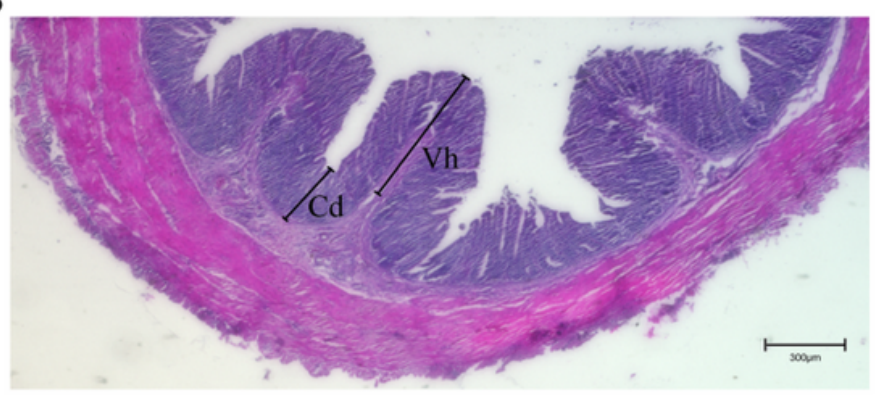

$\mathrm{D}$

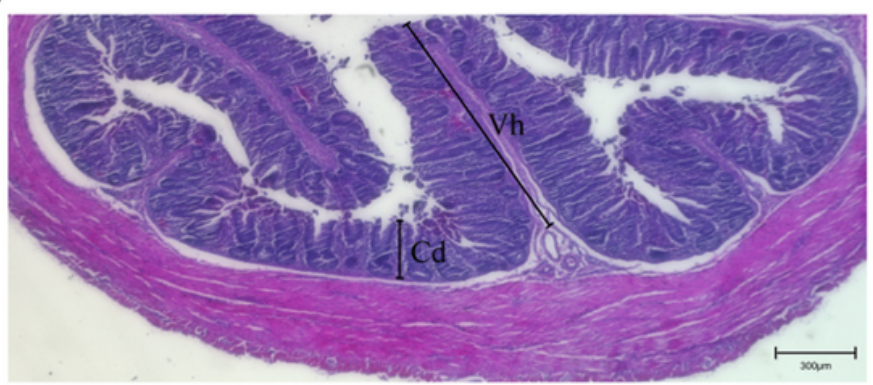

\section{Figure 1}

Histomorphology of chicken ileum and cecum. A, B) lleum and cecum from chickens at $42 \mathrm{~d}$ fed with the control diet. C, D) lleum and cecum from chickens at $42 \mathrm{~d}$ fed with diet supplemented with $1 \%$ Lasia spinosa Thw.. Bar=300 $\mu \mathrm{m}$. Vh: villus height; Cd: crypt depth. 
A

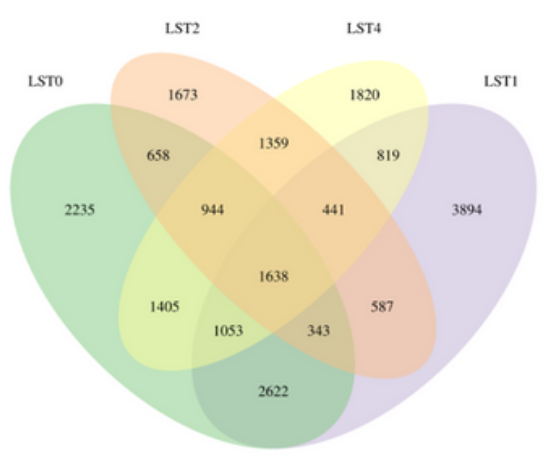

C

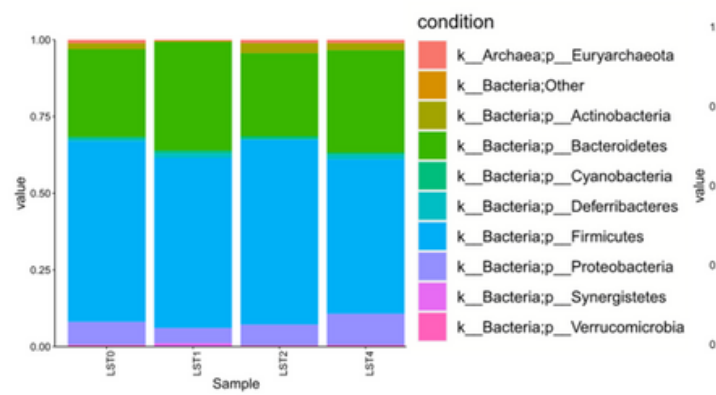

B

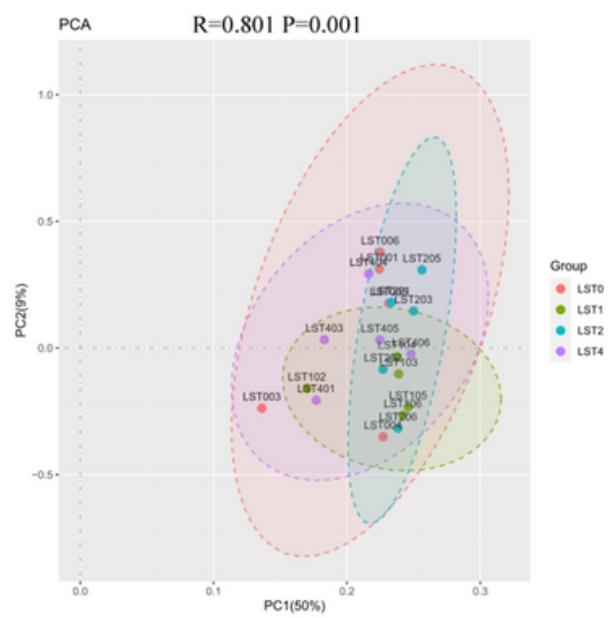

D

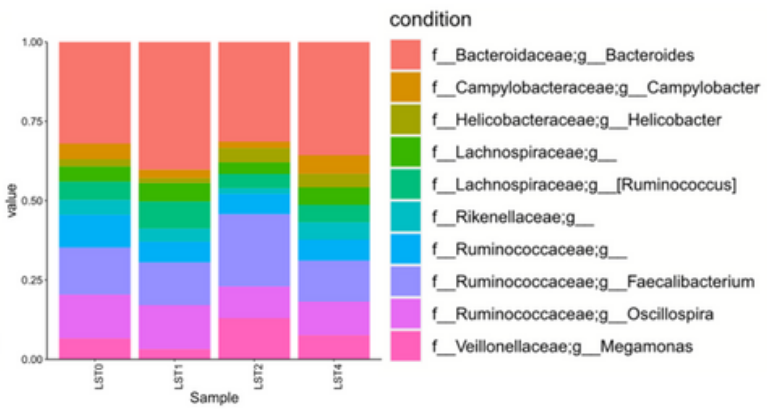

E
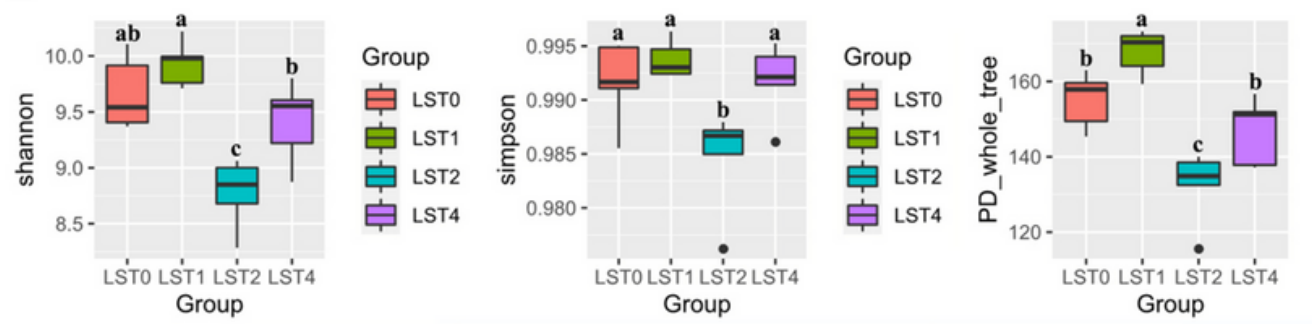

Group

追 LSTo

追 LST1

官 LST2

皁 LST4
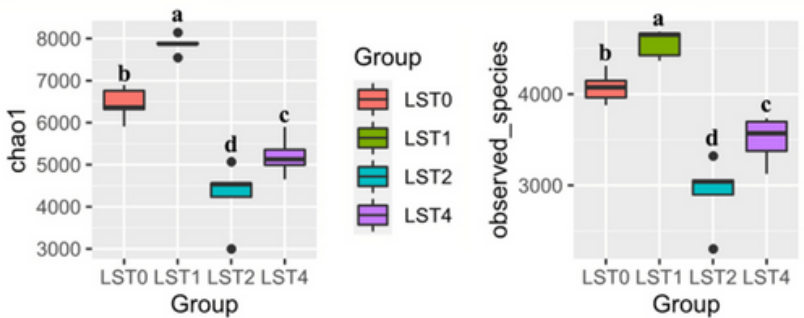

Group

官 LSTO

官 LST1

官 LST2

官 LST4

\section{Figure 2}

Abundance and diversity of microbial community in cecal contents of yellow-feathered broilers after dietary supplementation of LST powder for 42 days. A) The Venn diagram summarizing the numbers of common and unique OTUs in the microflora community. B) The PCA plot about the cecal microflora. C) and D) Microbial composition at the phylum and genus level. E) a-diversity about the cecal microbial community. a-d Boxes followed by different letters differ significantly $(P<0.05)$, followed by no or same letters indicated no significant difference $(P>0.05)$. LST: Lasia spinosa Thw.; LST0: control diet; LST1: 1\% supplementation level 
of Lasia spinosa Thw. powder; LST2: 2\% supplementation level of Lasia spinosa Thw. powder; LST4: 4\% supplementation level of Lasia spinosa Thw. powder.

A

\section{Cladogram}

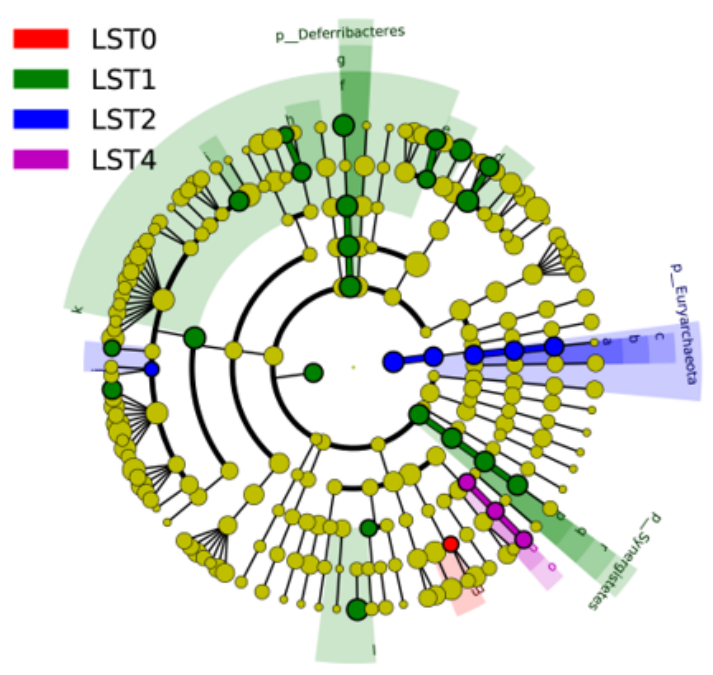

B

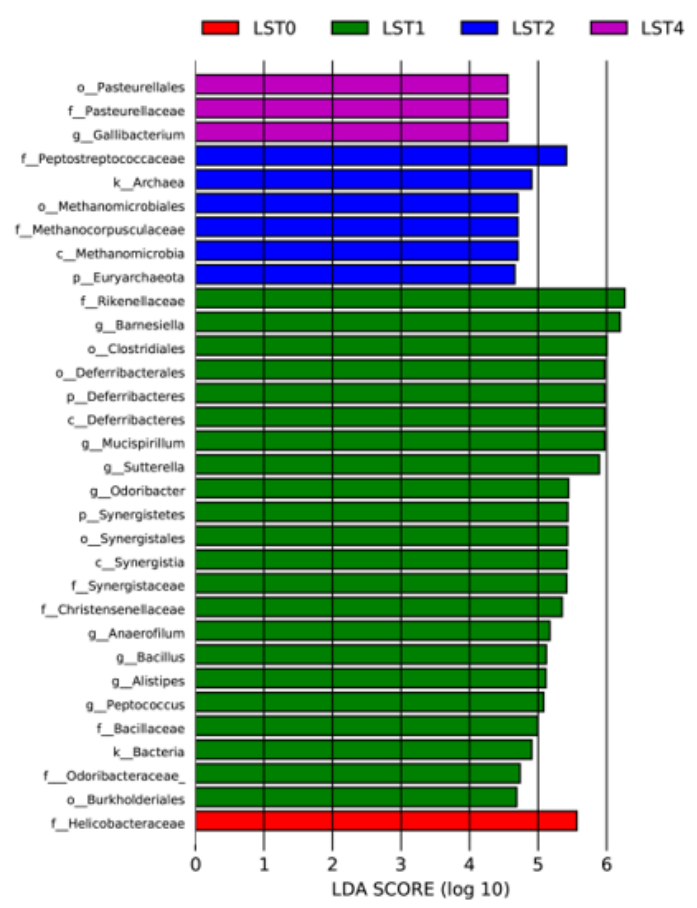

Figure 3

LEfSe (Linear discriminant analysis Effect Size) of cecal microbiota in yellow-feathered broilers fed with LST powder at day 42. A) Phylogenetic profile of specific bacterial taxa and predominant bacteria among the four different groups, as determined using the LEfSe analysis. Biomarker taxa are heighted by colored circles and shaded areas. Each circle's diameter is relative to abundance of taxa in the community. B) Histogram of LDA score computed for differential abundant taxa with cut-of LDA score >2.0. LST: Lasia spinosa Thw.; LST0: control diet; LST1: $1 \%$ supplementation level of Lasia spinosa Thw. powder; LST2: $2 \%$ supplementation level of Lasia spinosa Thw. powder; LST4: 4\% supplementation level of Lasia spinosa Thw. powder.

\section{Supplementary Files}

This is a list of supplementary files associated with this preprint. Click to download.

- Attachments.docx 